Check for updates

Cite this: Mater. Adv., 2021, 2, 7939

Received 17th June 2021, Accepted 19th October 2021

DOI: $10.1039 / \mathrm{d} 1 \mathrm{ma00526j}$

rsc.li/materials-advances

\title{
Single crystal growth and structural, magnetic, and magnetoelectric properties in spin-frustrated bow-tie lattice of $\alpha-\mathrm{Cu}_{5} \mathrm{O}_{2}\left(\mathrm{SeO}_{3}\right)_{2} \mathrm{Cl}_{2} \dagger$
}

\author{
D. Chandrasekhar Kakarla, (D) *ab Z. H. Yang, ${ }^{a}$ H. C. Wu, ${ }^{a}$ T. W. Kuo, ${ }^{a}$ Ajay Tiwari, ${ }^{a}$ \\ W.-H. Li, ${ }^{c}$ C. H. Lee, ${ }^{c}$ Y.-Y. Wang, ${ }^{d}$ J.-Y. Lin, ${ }^{\text {de }}$ C. K. Chang, ${ }^{f}$ B. H. Chen, \\ Chin-Wei Wang, id ${ }^{f}$ C. A. Lee, ${ }^{\text {bg }}$ Mitch M. C. Chou ${ }^{\text {bg }}$ and H. D. Yang (D) *ab
}

\begin{abstract}
Single crystals of $\alpha-\mathrm{Cu}_{5} \mathrm{O}_{2}\left(\mathrm{SeO}_{3}\right)_{2} \mathrm{Cl}_{2}$ are successfully prepared by the chemical vapor transport method, and their structural, magnetic, thermal, and dielectric properties are investigated as a function of temperature and magnetic field. Magnetization and specific heat measurements indicate a long-range antiferromagnetic transition at $T_{N}=32 \mathrm{~K}$. The spin ordering with a propagation vector $q=\left(\begin{array}{lll}1 / 2 & 0 & 0\end{array}\right)$ below $T_{N}$ is determined from the neutron diffraction patterns. The dielectric data reveal an anomaly near $T_{\mathrm{N}}$. Magnetic field-dependent dielectric measurements below $T_{\mathrm{N}}=32 \mathrm{~K}$ suggest a notable magnetodielectric effect with the scaling of magnetodielectric $\propto M^{2}$. Additionally, two more dielectric and associated specific heat anomalies are noticed above $T_{\mathrm{N}}$, collaborating with the synchrotron $x$-ray diffraction studies. These studies provide evidence that the bow-tie lattice of $\alpha-\mathrm{Cu}_{5} \mathrm{O}_{2}\left(\mathrm{SeO}_{3}\right)_{2} \mathrm{Cl}_{2}$ is a new magnetoelectric material.
\end{abstract}

\section{Introduction}

Multiferroics are extensively investigated in the field of condensed matter physics owing to their potential applications in spintronics and low-power-based microelectronics. ${ }^{1}$ The field of multiferroics is still evolving as the search for new multiferroics with novel mechanisms continues. Among several types of multiferroic materials, spin-induced ferroelectrics are of particular interest owing to their complex magnetic ground states and associated ferroelectric polarization. ${ }^{2}$ Numerous spin-induced type-II multiferroics have been explored on the basis of two origins: the inverse Dzyaloshinskii-Moriya (DM) interaction or exchange-striction mechanism. ${ }^{2}$ Among multiferroic materials, $\mathrm{Cu}^{2+} S=1 / 2$ spin-based multiferroics are garnering interest because of their complex magnetic ordering

\footnotetext{
${ }^{a}$ Department of Physics, National Sun Yat-sen University, Kaohsiung, 80424, Taiwan.E-mail: sekhar.kakarla@gmail.com,yang@mail.nsysu.edu.tw

${ }^{b}$ Center of Crystal Research, National Sun Yat-sen University, Kaohsiung, 80424, Taiwan

${ }^{c}$ Department of Physics, National Central University, Jhongli 32001, Taiwan

${ }^{d}$ Institute of Physics, National Chiao Tung University, Hsinchu 30010, Taiwan

${ }^{e}$ Center for Emergent Functional Matter Science, National Chiao Tung University, Hsinchu 30010, Taiwan

${ }^{f}$ National Synchrotron Radiation Research Center, Hsinchu, 30076, Taiwan

${ }^{g}$ Department of Materials and Optoelectronic Science, National Sun Yat-sen University, Kaohsiung, 80424, Taiwan

† Electronic supplementary information (ESI) available. See DOI: 10.1039/d1ma00526j
}

with spin frustration, which triggers various quantum effects. ${ }^{3-7}$ Many new physical properties have been discovered in cupric oxides, for example, superconductivity, charge density waves, multiferroicity, and skyrmions properties., ${ }^{3,4,6-10}$ When $\mathrm{Cu}^{2+}$ spin $1 / 2$ ions appear in a dimensionally restricted crystallographic lattice, they exhibit novel quantum magnetic landscapes. ${ }^{11-13}$ Lowdimension lattices consist either of quasi-two-dimensional (2D) layers in the form of Kagomé, pyrochlore, square, triangle, and honeycomb or quasi-one-dimensional spin-chains, spin-dimers, and spin-ladders are hosts for various interesting quantum phenomena, including the Bose-Einstein condensation, spinglass state, spin-Peierls transition, quantum spin-liquid state, and multiferroicity. ${ }^{5,11,14-18}$ In many $\mathrm{Cu}^{2+} S=1 / 2$ compounds, the spin frustration lattice with the competing magnetic exchange interaction between intralayers (chains) and interlayers (chains) invokes a fluctuation-induced quantum multiferroic behavior. $^{3}$

Thus far, spin $\mathrm{Cu}^{2+}$ spin $1 / 2$ systems with quasi-onedimensional spin chains, such as $\mathrm{LiCuVO}_{4}, \mathrm{GeCu}_{2} \mathrm{O}_{4}, \mathrm{Rb}_{2} \mathrm{Cu}_{2}$ $\mathrm{Mo}_{3} \mathrm{O}_{12}, \mathrm{CuCrO}_{2}, \mathrm{LiCu}_{2} \mathrm{O}_{2}, \mathrm{SrCuTe}_{2} \mathrm{O}_{6}$, and $\mathrm{CuCrO}_{4}$, have demonstrated multiferroicity. ${ }^{3,4,14,19-21}$ The competition between intra- and inter-chain magnetic exchange interactions results in nontrivial spin states, such as helical or conical, and invokes spontaneous electrical polarization. ${ }^{3,4,14,19-22}$ In addition to oxides, systems with halides and oxyhalides exhibiting spin spiral-driven multiferroics are gaining interest. For example, $\mathrm{CuCl}_{2}$ and $\mathrm{CuBr}_{2}$ exhibit multiferroicity at $T_{\mathrm{N}}=24$ and $90 \mathrm{~K}$, respectively. ${ }^{23,24}$ 
Table 1 List of known multiferroic and magnetoelectric compounds from $\mathrm{CuO}-\mathrm{CuCl}_{2}-\mathrm{SeO}_{2}$ ternary phase diagram

\begin{tabular}{lllll}
\hline Sample & Structure & Space group & Magnetic order & Electric order \\
\hline $\mathrm{CuO}$ & Monoclinic & $C 2 / c(15)$ & $T_{\mathrm{N}}=213-230 \mathrm{~K}$ & $T_{\mathrm{E}}=213-230 \mathrm{~K}$ \\
$\mathrm{CuCl}_{2}$ & Monoclinic & $C 2 / m(12)$ & $T_{\mathrm{N}}=24 \mathrm{~K}$ & $T_{\mathrm{E}}=24 \mathrm{~K}$ \\
$\mathrm{CuSeO}_{3}$ & orthorhombic & $P n m a(62)$ & $T_{\mathrm{C}}=25 \mathrm{~K}$ & $T_{\mathrm{E}}=25 \mathrm{~K}$ \\
$\mathrm{Cu}_{2} \mathrm{OCl}_{2}$ & Orthorhombic & $F d d d(70)$ & $T_{\mathrm{N}}=70 \mathrm{~K}$ & $T_{\mathrm{E}}=75 \mathrm{~K}$ \\
$\mathrm{Cu}_{2} \mathrm{OSeO}_{3}$ & Cubic & $P 2_{1} 3(198)$ & $T_{\mathrm{C}}=60 \mathrm{~K}$ & 32 \\
$\mathrm{Cu}_{9} \mathrm{O}_{2}\left(\mathrm{SeO}_{3}\right)_{4} \mathrm{Cl}_{6}$ & Monoclinic & $P 2_{1} / c(14)$ & $T_{\mathrm{N}}=37 \mathrm{~K}$ & 38,34 and 35 \\
$\mathrm{Cu}_{5} \mathrm{O}_{2}\left(\mathrm{SeO}_{3}\right)_{2} \mathrm{Cl}_{2}$ & Monoclinic & $P 2_{1} / c(14)$ & $T_{\mathrm{N}}=32 \mathrm{~K}$ & $T_{\mathrm{E}}=267 \mathrm{~K}$ \\
& & &
\end{tabular}

Along with halides, if the crystal structure contains chemical scissor lone-pair elements, such as $\mathrm{Se}^{4+}$ and $\mathrm{Te}^{4+}$ ions, will result in anisotropic magnetic and multiferroic behaviors. ${ }^{12,25} \mathrm{~A}$ lowdimensional crystal structure with anisotropic spin-induced multiferroicity was reported in the magnetic layers of a $\mathrm{Cu}_{3} \mathrm{Bi}(-$ $\left.\mathrm{SeO}_{3}\right)_{2} \mathrm{O}_{2} \mathrm{Cl}_{2}$ system. ${ }^{26}$ In this context, compounds from the $\mathrm{CuO}-\mathrm{CuCl}_{2}-\mathrm{SeO}_{2}$ ternary phase diagram have been reported to exhibit exotic quantum magnetic states such as skyrmions, along with unique multiferroic properties, ${ }^{8,24,27-38}$ as shown in Table 1.

$\mathrm{Cu}_{5} \mathrm{O}_{2}\left(\mathrm{SeO}_{3}\right)_{2} \mathrm{Cl}_{2}$ appeared in two polymorphic phases: georgbokiite $\left[\alpha-\mathrm{Cu}_{5} \mathrm{O}_{2}\left(\mathrm{SeO}_{3}\right)_{2} \mathrm{Cl}_{2}\right.$ : dark brown color] and parageorgbokiite $\left[\beta-\mathrm{Cu}_{5} \mathrm{O}_{2}\left(\mathrm{SeO}_{3}\right)_{2} \mathrm{Cl}_{2}\right.$ : green color]; natural mineral species occurred at the second cinder cone of the Great Tolbachik Fissure Eruption, Kamchatka Peninsula, Russia. ${ }^{39-41}$ Because $\alpha-\mathrm{Cu}_{5} \mathrm{O}_{2}\left(\mathrm{SeO}_{3}\right)_{2} \mathrm{Cl}_{2}$ exists in the $\mathrm{CuO}-\mathrm{CuCl}_{2}-\mathrm{SeO}_{2}$ phase diagram, its physical properties are worth investigating. We synthesized $\alpha-\mathrm{Cu}_{5} \mathrm{O}_{2}\left(\mathrm{SeO}_{3}\right)_{2} \mathrm{Cl}_{2}$ single crystals using the chemical vapor transport method and investigated their magnetic and magnetodielectric properties with a view to adding a new member of magnetoelectric material to the $\mathrm{CuO}-\mathrm{CuCl}_{2}-\mathrm{SeO}_{2}$ family.

\section{Experimental details}

\subsection{Single crystal and polycrystalline growth}

Single crystals of $\alpha-\mathrm{Cu}_{5} \mathrm{O}_{2}\left(\mathrm{SeO}_{3}\right)_{2} \mathrm{Cl}_{2}$ were grown using a standard chemical vapor transport phase method. High-purity $\mathrm{CuO}$ (Alfa-Aesar, 99.995\%), $\mathrm{CuCl}_{2}$ (Alfa-Aesar, 99.995\%), and $\mathrm{SeO}_{2}$ (Alfa-Aesar, 99.999\%) powders in a molar ratio of $3: 2: 2$ were mixed under Ar atmosphere. A $15 \mathrm{~g}$ sample was loaded into a quartz ampule with $100 \mathrm{~mm}$ length and $15 \mathrm{~mm}$ diameter. The ampule was horizontally placed in a tubular two-zone furnace and then heated at a rate of $0.5{ }^{\circ} \mathrm{C} \mathrm{min}^{-1}$. As shown in Fig. 1(a), for the growth of $\alpha-\mathrm{Cu}_{5} \mathrm{O}_{2}\left(\mathrm{SeO}_{3}\right)_{2} \mathrm{Cl}_{2}$ single crystals, the optimum temperatures at the source and deposition zones were set to $500{ }^{\circ} \mathrm{C}$ and $450{ }^{\circ} \mathrm{C}$, respectively, for 15 days. Subsequently, the temperature was reduced to room temperature at a rate of $0.5{ }^{\circ} \mathrm{C} \mathrm{min}{ }^{-1}$. Several brownish-colored $\alpha-\mathrm{Cu}_{5} \mathrm{O}_{2}\left(\mathrm{SeO}_{3}\right)_{2} \mathrm{Cl}_{2}$ single crystals, along with an unidentified powder residue, appeared in the hot zone; $\alpha-\mathrm{Cu}_{5} \mathrm{O}_{2}\left(\mathrm{SeO}_{3}\right)_{2} \mathrm{Cl}_{2}$ single crystals were selected on the basis of their color and appearance. Green, thin, platelet-like, $\mathrm{Cu}_{9} \mathrm{O}_{2}\left(\mathrm{SeO}_{3}\right)_{4} \mathrm{Cl}_{6}$ single crystals $(2-3 \mathrm{~mm}$ in length) were formed at the cold end. Electron probe microanalysis (EPMA) [shown in Fig. S1 and Table S1, ESI $\dagger$ ] revealed that the stoichiometry of all the heavy elements in the synthesized products was similar to that expected in $\alpha-\mathrm{Cu}_{5} \mathrm{O}_{2}\left(\mathrm{SeO}_{3}\right)_{2} \mathrm{Cl}_{2}$.

\subsection{Characterization}

The quality of the grown $\alpha-\mathrm{Cu}_{5} \mathrm{O}_{2}\left(\mathrm{SeO}_{3}\right)_{2} \mathrm{Cl}_{2}$ single crystals was determined by high-resolution synchrotron X-ray diffraction (SXRD) using the TPS 09A beamline of the Taiwan Photon Source at the National Synchrotron Radiation Research Center. For the SXRD measurements, single crystals were ground to a fine powder. A closed-cycle cryostat was used to control the temperature during the measurements. The samples were mounted on a glass tube, and data were collected in reflection geometry using a wavelength of $\lambda=0.61922 \AA$ with a double crystal monochromator $\mathrm{Si}(111)$ with an energy resolution of $1.33 \times 10^{-4}$. Diffraction data were collected at $2^{\circ}-120^{\circ}$ at intervals of $0.004^{\circ}$. Rietveld refinement was performed using the FullProf suite software. The crystal structure was drawn using the VESTA software (version 3.5.2). DC magnetic measurements were conducted using a Quantum Design magnetic

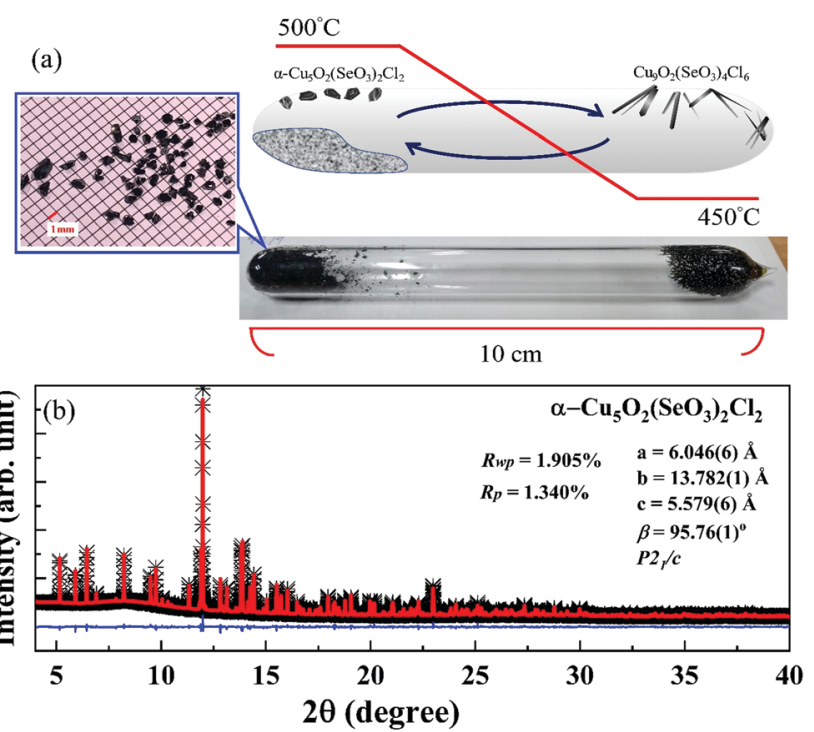

Fig. 1 (a) Experimental two-zone growth of $\alpha-\mathrm{Cu}_{5} \mathrm{O}_{2}\left(\mathrm{SeO}_{3}\right)_{2} \mathrm{Cl}_{2}$ single crystals; pictorial representation of quartz ampoule and a photograph of the real quartz ampoule. Red line in the figure denotes the temperatures of the two-zone furnace's hot and cold ends. Brownish $\alpha-\mathrm{Cu}_{5} \mathrm{O}_{2}\left(\mathrm{SeO}_{3}\right)_{2} \mathrm{Cl}_{2}$ and green $\mathrm{Cu}_{9} \mathrm{O}_{2}\left(\mathrm{SeO}_{3}\right)_{4} \mathrm{Cl}_{6}$ single crystals grown at the hot and cold ends, respectively, are shown in (a). Light microscopic images of grown $\alpha-\mathrm{Cu}_{5} \mathrm{O}_{2}\left(\mathrm{SeO}_{3}\right)_{2} \mathrm{Cl}_{2}$ single crystals at quartz ampoule hot zone. (b) Highresolution synchrotron $X$-ray diffraction data along with Rietveld refinement of $\alpha-\mathrm{Cu}_{5} \mathrm{O}_{2}\left(\mathrm{SeO}_{3}\right)_{2} \mathrm{Cl}_{2}$ at $300 \mathrm{~K}$. 
property measurement system (MPMS-XL7). Temperaturedependent specific heat $C(T)$ measurements were performed on a plate-like single crystal using a heat-pulsed thermal relaxation calorimeter in the temperature range of $2-300 \mathrm{~K}$ and in magnetic fields of up to $H=9 \mathrm{~T}$. Neutron powder diffraction measurements were performed on a high-intensity powder diffractometer WOMBAT, ${ }^{42}$ and a cold neutron triple-axis spectrometer SIKA at the Bragg Institute, ANSTO. Diffraction measurements were performed on the WOMBAT diffractometer using an incident wavelength of $\lambda=4.78 \AA$ ( $3.58 \mathrm{meV})$ defined by pyrolytic graphite (PG)(002) crystals at the monochromator position, with a take-off angle of $90^{\circ}$. SIKA was operated in the diffraction mode, where the energy of the neutrons was defined by $\mathrm{PG}(002)$ crystals at both the monochromator and analyzer positions using fixed final energy of $3 \mathrm{meV}$, and a Be filter was used to suppress the higher-order contaminations. For the neutron diffraction measurements, approximately $2 \mathrm{~g}$ of the powdered sample was loaded into an

Table 2 Lattice parameters, atomic positions, and reliability factors of $\alpha-\mathrm{Cu}_{5} \mathrm{O}_{2}\left(\mathrm{SeO}_{3}\right)_{2} \mathrm{Cl}_{2}$ at $T=300 \mathrm{~K}$ obtained by assuming space group $P 2_{1} / \mathrm{C}$

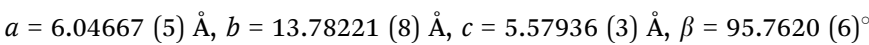

\begin{tabular}{llrlll}
\hline Atom & $x$ & \multicolumn{1}{l}{$y$} & $z$ & $B_{\text {iso }}\left(\AA^{2}\right)$ & Occupancy \\
\hline $\mathrm{Cu} 1$ & $0.4343(5)$ & $0.1900(1)$ & $0.5553(7)$ & 0.219 & 1 \\
$\mathrm{Cu} 2$ & $0.5000(0)$ & $0.0000(0)$ & $1.0000(0)$ & 0.256 & 1 \\
$\mathrm{Cu} 3$ & $0.2572(4)$ & $-0.0035(9)$ & $0.4859(4)$ & 0.259 & 1 \\
$\mathrm{Se}$ & $0.0624(2)$ & $0.1351(4)$ & $0.8980(5)$ & 0.444 & 1 \\
$\mathrm{O} 1$ & $0.5037(5)$ & $0.0632(4)$ & $0.6795(2)$ & 0.545 & 1 \\
O2 & $0.2230(4)$ & $0.0583(3)$ & $1.0073(5)$ & 0.152 & 1 \\
O3 & $0.0391(7)$ & $0.0654(4)$ & $0.6390(4)$ & 1.101 & 1 \\
O4 & $0.2520(6)$ & $0.2194(1)$ & $0.8083(5)$ & 2.485 & 1 \\
Cl & $0.6712(1)$ & $0.1760(6)$ & $0.2703(3)$ & 2.475 & 1
\end{tabular}

$\chi^{2}=6.04, R_{\mathrm{p}}=1.34 \%, \mathrm{w} R_{\mathrm{p}}=1.905 \%$. aluminum sample holder filled with helium gas to facilitate thermal conduction. The sample temperature was controlled using a closed-cycle cryocooler, operating between 3 and $60 \mathrm{~K}$. To investigate the dielectric properties of $\alpha-\mathrm{Cu}_{5} \mathrm{O}_{2}\left(\mathrm{SeO}_{3}\right)_{2} \mathrm{Cl}_{2}$, a single crystal measuring $\sim 2 \mathrm{~mm}$ was selected. Silver paint was employed as electrodes on both sides of the crystal to form a parallel-plate capacitor. Dielectric $\left(\varepsilon^{\prime}\right)$ measurements were performed using an Agilent E4980A precision LCR meter with an excitation voltage of $5 \mathrm{~V}$. High-temperature $\varepsilon^{\prime}$ measurements were performed using a closed-cycle cryostat. For magnetodielectric (MD) and pyrocurrent measurements, a homemade sample probe was inserted into the Quantum design MPMS system.

\section{Results and discussion}

\subsection{Structural analysis}

High-resolution SXRD is effective for investigating minute secondary phases. As shown in Fig. 1(b), all the reflections in the SXRD pattern collected at room temperature were assigned to the $\alpha-\mathrm{Cu}_{5} \mathrm{O}_{2}\left(\mathrm{SeO}_{3}\right)_{2} \mathrm{Cl}_{2}$ phase without any secondary phase. The Rietveld refinement of the high-resolution SXRD data confirmed a monoclinic crystal structure with the space group $P 2_{1} / c$. The obtained refinement parameters and unit cell parameters (see Table 2), were consistent with those in previous studies. $^{12,40}$

The crystal structure drawn from the output files of the SXRD refinement using the VESTA software are shown in Fig. 2, with a description of the $\mathrm{Cu}$ ions in the polyhedral representation. The monoclinic $\alpha-\mathrm{Cu}_{5} \mathrm{O}_{2}\left(\mathrm{SeO}_{3}\right)_{2} \mathrm{Cl}_{2}$ unit cell comprised three different $\mathrm{Cu}$ atoms, situated in polyhedra formed by the combination of $\mathrm{Cl}$ and $\mathrm{O}$ anions. In the $a-c$ plane [see Fig. 2(a)],

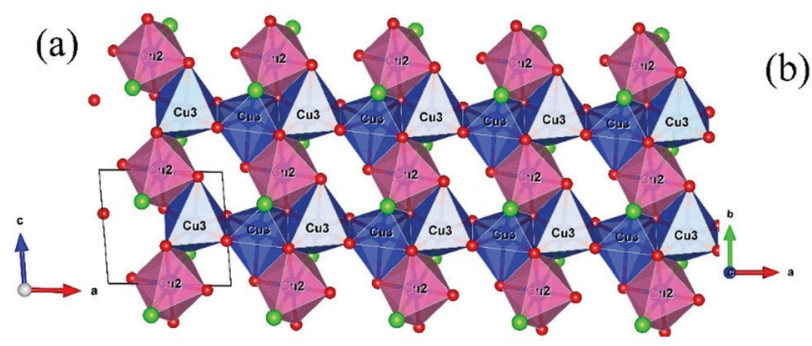

(b)
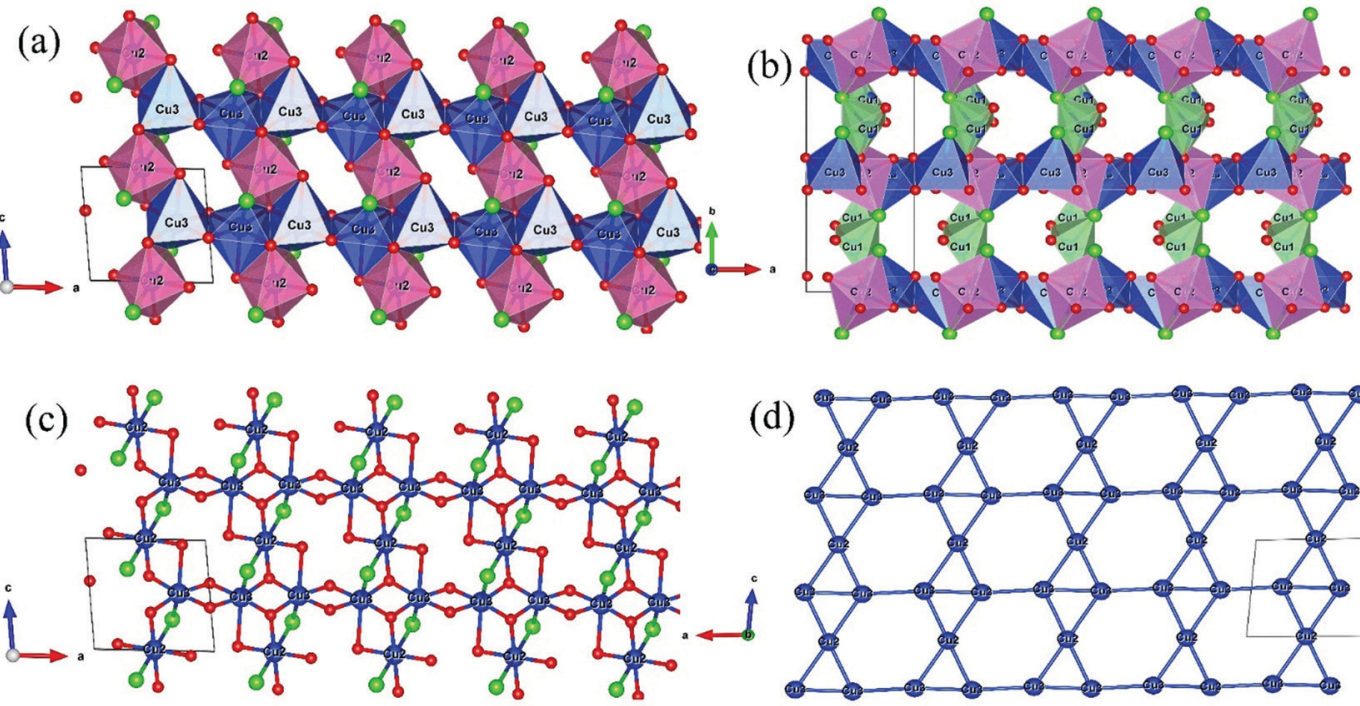

(d)

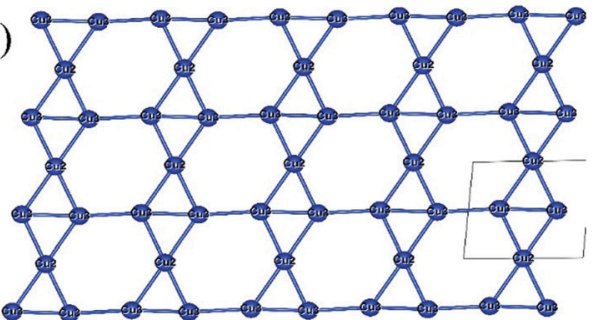

Fig. 2 Crystal structure of $\alpha-\mathrm{Cu}_{5} \mathrm{O}_{2}\left(\mathrm{SeO}_{3}\right)_{2} \mathrm{Cl}_{2}$ single crystal in different directions (a) $a-c$ plane; (b) $a-b$ plane. Cu2 and Cu3 atoms occupied the octahedral and Cu1 occupied square pyramid coordination; for clarity, Se atoms are omitted from the figures. $\mathrm{Cu} 3 \mathrm{O}_{5} \mathrm{Cl}_{1} \mathrm{Cu} 2 \mathrm{O}_{4} \mathrm{Cl}_{2}$, and $\mathrm{Cu} 3 \mathrm{O}_{3} \mathrm{Cl}_{2}$ formed blue, pink, and green polyhedra, respectively; a-c plane (c) without polyhedral and (d) only Cu atoms; a bow-tie lattice formed by $\mathrm{Cu} 2$ and Cu3 atoms in a-c plane. 
the $\mathrm{Cu} 2 \mathrm{O}_{4} \mathrm{Cl}_{2}$ and $\mathrm{Cu} 3 \mathrm{O}_{5} \mathrm{Cl}$ octahedra formed alternating layers along the $a$-axis connected via the sharing of octahedral edges. The $\mathrm{Cu} 2$ has a square $\mathrm{Cu}_{2} \mathrm{O}_{4}$ basal plane with $\mathrm{Cu}-\mathrm{O}$ bond distances ranging from 1.945 to $1.991 \AA$, whereas the two $\mathrm{Cl}$ atoms were located at the apex with a longer bonding distance of $2.986 \AA$. This significant difference between the atomic lengths of the basal and apex axes was due to the Jahn-Teller distortion of the $\mathrm{Cu}^{2+}$ ions. ${ }^{41}$ The $\mathrm{Cu} 3$ octahedron was formed by the $\mathrm{Cu}_{3} \mathrm{O}_{4}$ basal plane (basal Cu3-O distance of 1.898-2.043 $\AA$ ) along the distorted apex axes, one O (Cu3-O distance of $2.423 \AA$ ) , and one $\mathrm{Cl}(\mathrm{Cu} 3-\mathrm{Cl}$ distance of $2.751 \AA$ ) ions.

The alternating $a-c$ planes [see Fig. 2(b)] were linked along the $b$-axis via two edges of the $\mathrm{Cu}_{1} \mathrm{O}_{3} \mathrm{Cl}_{2}$ square pyramid polyhedral with a $\mathrm{Cu}_{1} \mathrm{O}_{3} \mathrm{Cl}$ square base (Cu1-O distance of 1.912-2.09 $\AA$ and Cu1-Cl distance of $2.253 \AA$ ) and one $\mathrm{Cl}$ atom (Cu1-Cl distance of $2.560 \AA$ ) at the apex point. The Se atoms formed a one-sided three-fold coordination polyhedral connecting to the Cu-polyhedra by corner- and edge-sharing, but were isolated from each other. Fig. 2(c) shows the two-dimensional $a-c$ plane, without a polyhedral representation. The $\mathrm{Cu} 2$ and $\mathrm{Cu} 3$ atoms linked chains appeared along the $a$-axis. The edgesharing $\mathrm{Cu}_{3} \mathrm{O}_{4}$ square lattice appeared with alternating long (3.132 $\mathrm{\AA}$ ) and short (2.926 $\mathrm{\AA}) \mathrm{Cu} 3-\mathrm{Cu} 3$ bond lengths along the $a$-axis. Fig. 2(d) shows the bonding of $\mathrm{Cu}$ atoms in the $a-c$ plane; the $\mathrm{Cu}$ atoms form the edge, and corner-sharing distortive alternating triangular and honeycomb lattices called as bow-tie lattice, which can be regarded as between a honeycomb lattice and a Kagomé lattice. ${ }^{43}$ A similar network of magnetic ions was previously identified in $\mathrm{Na}_{2} \mathrm{Ni}_{3}(\mathrm{OH})_{2}\left(\mathrm{PO}_{4}\right)_{2} \cdot{ }^{43}$ The spins at bow-tie lattice is highly likely to induce spin-frustration in the $a-c$ plane. Furthermore, the alternating $\mathrm{Cu} 3-\mathrm{Cu} 3$ bond lengths along the $a$-axis may cause the spin structure to become more frustrated.

\subsection{Magnetic properties}

The temperature dependence of the static zero-field cooled (ZFC) and field-cooled (FC) magnetic susceptibility $(\chi=M / H)$ for $\alpha-\mathrm{Cu}_{5} \mathrm{O}_{2}\left(\mathrm{SeO}_{3}\right)_{2} \mathrm{Cl}_{2}$ in the range $5-320 \mathrm{~K}$ with an applied field of 1000 Oe is shown in Fig. 3(a). Owing to the small size and mass of the grown single crystals, magnetic measurements were performed on a collection of several single crystals (approximately $35.5 \mathrm{mg}$ ) with random orientations. The ZFCFC $\chi$ versus $T$ curves exhibited almost similar trends down to the lowest temperatures without any irreversibility. The $\chi$ versus $T$ curves increased with decreasing temperature and exhibited a broad hump at $T_{\text {SRMO }} \sim 97 \mathrm{~K}$; this is characteristic of short-range or low-dimensional magnetic ordering (SRMO) of several low-dimensional magnetic systems. ${ }^{6,29}$ Although the crystal structure showed the three-dimensional connection of $\mathrm{Cu}^{2+}$ spin $1 / 2$ ions, the presence of an anomaly at $T_{\text {SRMO }}$ suggested that the predominant magnetic exchange interactions occur at the two-dimensional $a-c$ plane, where $\mathrm{Cu} 2$ and $\mathrm{Cu} 3$ spins formed distorted bow-tie lattice. The $a-c$ planes were magnetically connected through a weak exchange interaction. At low temperatures, a kink appeared in the $\chi$ versus $T$ curves, indicating long-range antiferromagnetic ordering at $T_{\mathrm{N}} \sim 32 \mathrm{~K}$, as verified by the specific heat measurements shown in Fig. 4. The effective paramagnetic moment $\mu_{\text {eff }}=\sqrt{ }\left(3 k_{\mathrm{B}} c / N_{\mathrm{A}}\right)$ above $T_{\text {SRMO }}$ was estimated by fitting the Curie-Weiss $(\mathrm{C}-\mathrm{W})$ equation $\chi=\frac{C}{\left(T-\theta_{\mathrm{CW}}\right)}$, where $\theta_{\mathrm{CW}}$ and $k_{\mathrm{B}}$ denote the Curie temperature and Boltzmann constant, respectively. The fit of the data to the $\mathrm{C}-\mathrm{W}$ law in the temperature range of $250-320 \mathrm{~K}$ resulted in $\theta_{\mathrm{CW}}=-214.4 \mathrm{~K}$ and an effective magnetic moment $\mu_{\mathrm{eff}} \sim$ $2.18 \mu_{\mathrm{B}}$. The value of $\mu_{\text {eff }}$ was slightly higher than the expected paramagnetic $\mathrm{Cu}^{2+}$ spin-only value $\left(1.73 \mu_{\mathrm{B}}\right)$. This may be due to the estimated $\mu_{\mathrm{eff}}$ is close to $T_{\mathrm{SRMO}}$. A negative $\theta_{\mathrm{CW}}$ indicates a dominantly antiferromagnetic spin-exchange interaction. The spin frustration index was $\frac{T_{\mathrm{N}}}{\theta_{\mathrm{CW}}} \sim 0.133$, which is close to the frustration index limit $\left(\frac{T_{\mathrm{N}}}{\theta_{\mathrm{CW}}} \sim 0.1\right)$ of typical spin-frustrated systems. $^{44,45}$

The isothermal magnetization data $M(H)$, are shown in Fig. 4(b). The $M$ versus $H$ curves at different temperatures exhibited linearity without any hysteresis loops. The $\mathrm{d} M / \mathrm{d} H$ versus $H$ curves (not shown herein) below $T_{\mathrm{N}}$ did not exhibit a clear metamagnetic transition within $7 \mathrm{~T}$, signifying the robustness of the antiferromagnetic ground state of $\alpha-\mathrm{Cu}_{5} \mathrm{O}_{2}\left(\mathrm{SeO}_{3}\right)_{2} \mathrm{Cl}_{2}$.
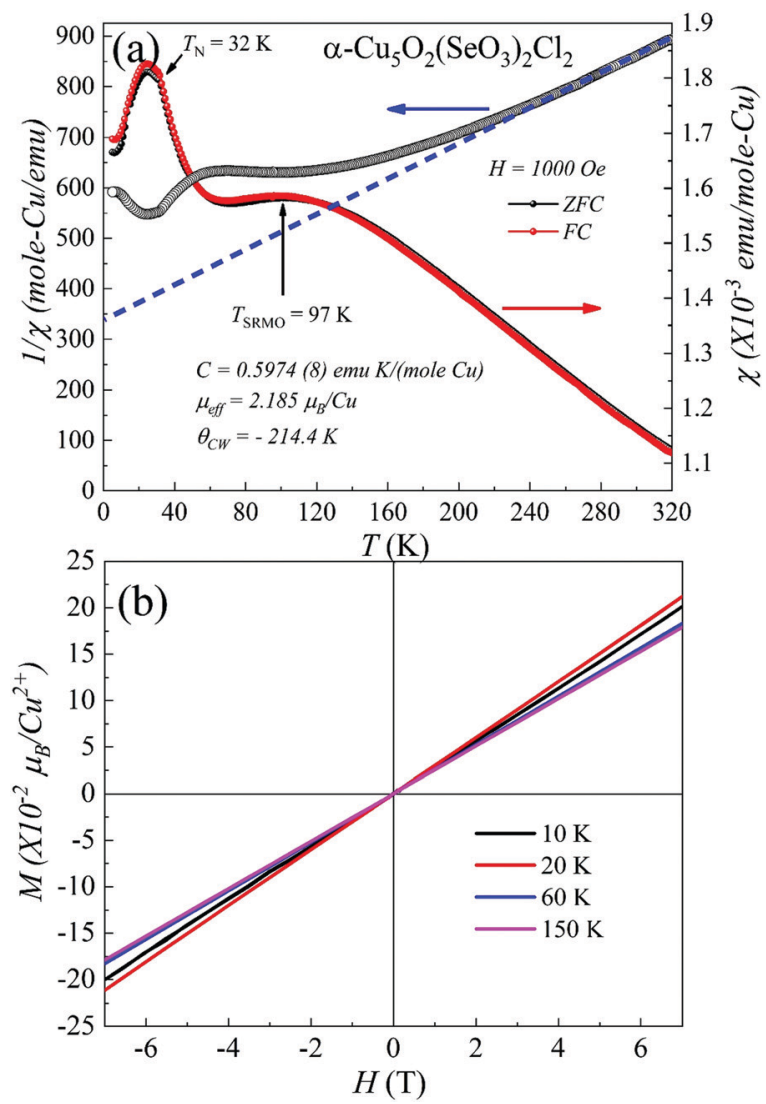

Fig. 3 (a) Variation in ZFC-FC susceptibility (right $y$-axis) and inverse susceptibility (left $y$-axis) of $\alpha-\mathrm{Cu}_{5} \mathrm{O}_{2}\left(\mathrm{SeO}_{3}\right)_{2} \mathrm{Cl}_{2}$ single crystal with $T$ in the $5-320 \mathrm{~K}$ range. Solid blue line through the inverse susceptibility data denotes the Curie-Weiss fit. (b) Isothermal $M$ vs. $H$ curves for different temperatures. 


\subsection{Specific-heat measurements}

The $C(T)$ of the $\alpha-\mathrm{Cu}_{5} \mathrm{O}_{2}\left(\mathrm{SeO}_{3}\right)_{2} \mathrm{Cl}_{2}$ single crystal as a function of temperature is shown in Fig. 4(a). The $C(T)$ exhibits a $\lambda$-type peak at $32 \mathrm{~K}$, justifying the assignment of a genuine long-range magnetic order at $T_{\mathrm{N}}$, as shown in Fig. 3(a). Furthermore, a closer observation of the $C(T)$ [see the inset of Fig. 4(a)] at high temperatures revealed clear slope changes at both $T=230$ and $280 \mathrm{~K}$. The origin of these signatures in $C(T)$ is closely associated with structural changes, as confirmed by high-resolution synchrotron diffraction and the dielectric constant $\varepsilon^{\prime}$ [see Fig. 8 and Fig. 9].

Under magnetic fields, interestingly, $H$ has only minimal effects on $C(T)$. For example, the shape of the $\lambda$-type peak at $T_{\mathrm{N}}$ [see in Fig. 4(b)] did not change with $H$ up to 9 T, indicating the robust nature of this antiferromagnetic order. These results are consistent with the linear $M-H$ curves up to $7 \mathrm{~T}$, as shown in Fig. 3(b). However, a few subtleties may appear in the magnetic properties at $H>7 \mathrm{~T}$. A close analysis revealed that $T_{\mathrm{N}}$ increased slightly when $H \geq 8 \mathrm{~T}$ compared with at $H=6 \mathrm{~T}$. This transition became even sharper when $H \geq 8 \mathrm{~T}$. Hence, the metamagnetic or other new magnetic states cannot be excluded at high $H$.

\subsection{Low-temperature spin structure}

We employed neutron powder diffraction to detect Bragg intensities from magnetic correlations. All the diffraction peaks
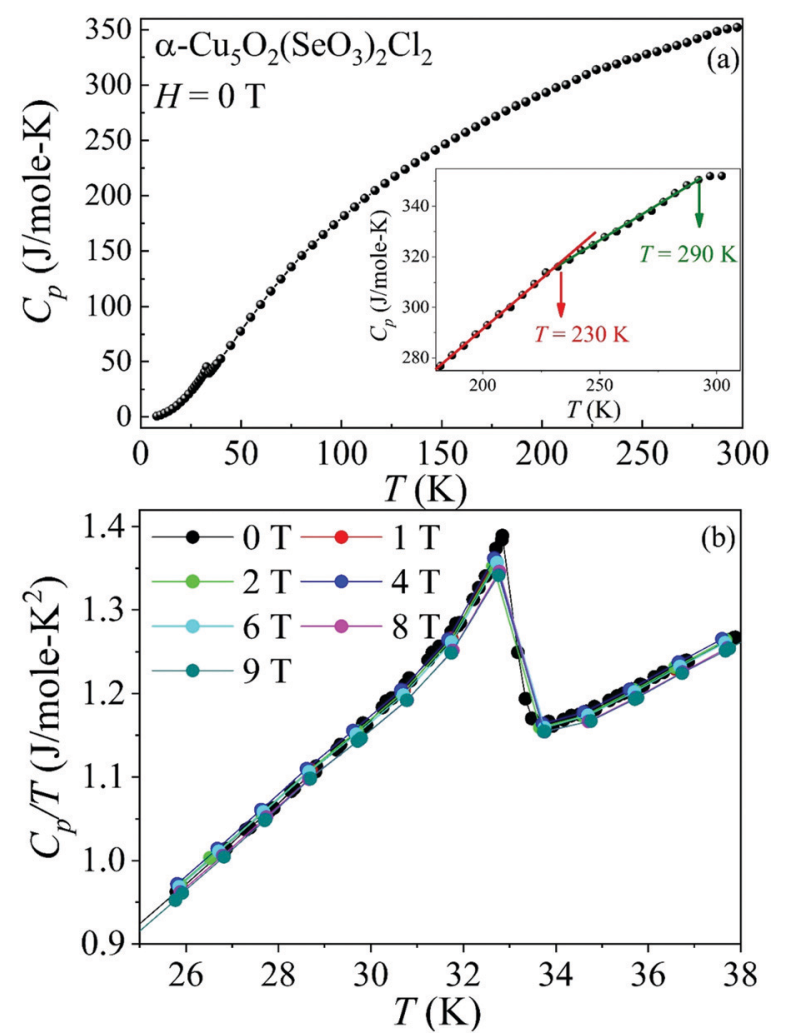

Fig. 4 (a) $\mathrm{C}_{\mathrm{p}}$ vs. $T$ curve for $\alpha-\mathrm{Cu}_{5} \mathrm{O}_{2}\left(\mathrm{SeO}_{3}\right)_{2} \mathrm{Cl}_{2}$ single crystal; inset shows $C_{p}$ vs. $T$ curves at high temperature; red and green lines indicate slope changes at 230 and $290 \mathrm{~K}$, respectively. (b) Variation in $C_{\mathrm{p}} / T$ vs. $T$ curves under different applied magnetic fields; peaks at $T_{N}$ appeared robust for field up to $9 \mathrm{~T}$. observed at $60 \mathrm{~K}$ can be described using the same monoclinic $P 2_{1} / c$ crystalline structure obtained from the XRD patterns [see Fig. 1(b)]. Several additional Bragg peaks appeared in the diffraction pattern at $3.5 \mathrm{~K}$. Fig. 5(a) shows the magnetic diffraction pattern at $3.5 \mathrm{~K}$; this was obtained by calculating the difference between the 3.5 and $60 \mathrm{~K}$ patterns, in order to isolate the magnetic intensities that developed upon cooling from 60 to $3.5 \mathrm{~K}$. All the magnetic reflections may be indexed using a propagation vector of $q=\left(\begin{array}{lll}1 / 2 & 0 & 0\end{array}\right)$, revealing that the magnetic unit cell for the $\mathrm{Cu}$ spins is double the nuclear one along the crystallographic $a$-axis direction. A symmetry analysis based on the BasIreps program yielded four allowed irreducible representations, namely, $\Gamma_{1}, \Gamma_{2}, \Gamma_{3}$, and $\Gamma_{4}$, for the $P 2_{1} / c$ space group coupled to the $\left(\begin{array}{lll}1 / 2 & 0 & 0\end{array}\right)$ propagation vector. A magnetic diffraction pattern was analyzed using the FullProf program, ${ }^{46}$ in which the $P 2_{1} / c$ spatial symmetry of the crystalline structure was preserved, as for the magnetic structure. The $\Gamma_{1}$ and $\Gamma_{3}$ symmetries generated an intensity for the $\left(\begin{array}{lll}1 / 2 & 0 & 0\end{array}\right)$ reflection,
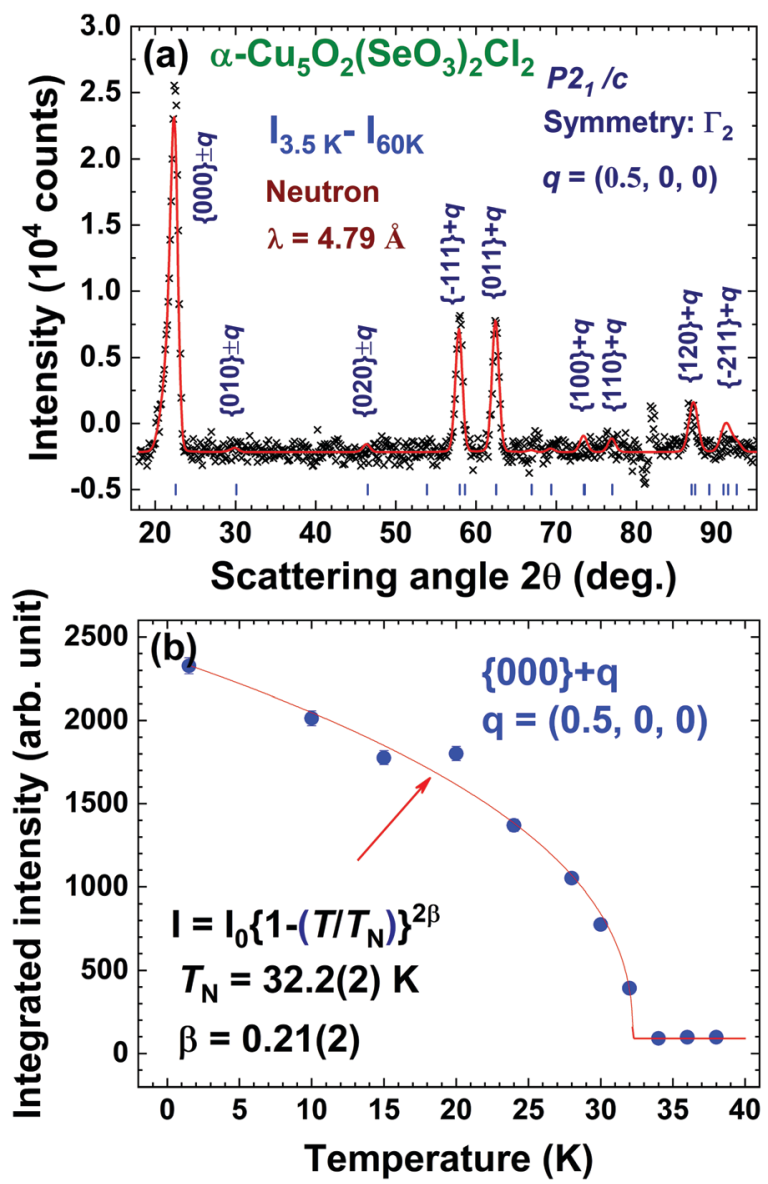

Fig. 5 (a) Observed (crosses) and calculated (solid lines) neutron magnetic diffraction patterns at $3.5 \mathrm{~K}$, assuming monoclinic $P 2_{1} / c$ magnetic symmetry for $\mathrm{Cu}$ spins. Magnetic reflections are indexed with propagation vector $q=\left(\begin{array}{lll}1 / 2 & 0 & 0\end{array}\right)$. Solid vertical lines below the pattern indicate the calculated Bragg positions of the proposed magnetic structure. (b) Temperature dependence of integrated intensity of $\{000\}+q$ reflection. Solid curve indicates results of data fitted to the expression list in the plot, yielding an antiferromagnetic transition temperature of $T_{\mathrm{N}}=32.2(3) \mathrm{K}$ and a critical exponent of $\beta=0.21(2)$ for the magnetic transition. 
which is comparable to that of the $\left(\begin{array}{lll}1 / 2 & 1 & 1\end{array}\right)$ reflection and zero moment of the $\mathrm{Cu}^{2+}$ ions. The $\Gamma_{4}$ symmetry generated a strong intensity for the $\left(\begin{array}{lll}1 / 2 & 1 & 0\end{array}\right)$ reflection or required a moment for the $\mathrm{Cu}_{3}$ ions, which was 2.2 times the moment for $\mathrm{Cu}^{2+}$ ions. $\Gamma_{2}$ exhibited time reversal in the $c$-glide plane to generate a magnetic diffraction pattern that best fitted the observed patterns. Fig. 6(a) shows the spin arrangement obtained from the fits to the $3.5 \mathrm{~K}$ magnetic pattern, assuming the $P 2_{1} / c$ spatial symmetry coupled to the $\Gamma_{2}$ magnetic symmetry. The magnetic diffraction pattern at $3.5 \mathrm{~K}$ can be effectively described [solid line in Fig. 5(a)] using the proposed spin structure shown in Fig. 6(a). The spin arrangement of the $\mathrm{Cu} 2$ and $\mathrm{Cu} 3$ ions, as shown in Fig. 6(b), can be viewed as the formation of antiferromagnetically coupled ferromagnetic-spin-dimers (dashed rectangular blocks) chains (blue arrows) along the crystallographic $a$-axis direction, which is separated by antiferromagnetic spin chains (red arrows). Antiferromagnetically coupled ferromagnetic dimers are intriguing. Along with the frustrating nature of the $\mathrm{Cu}$ sublattice, these dimers can yield exotic states at extremely low temperatures;
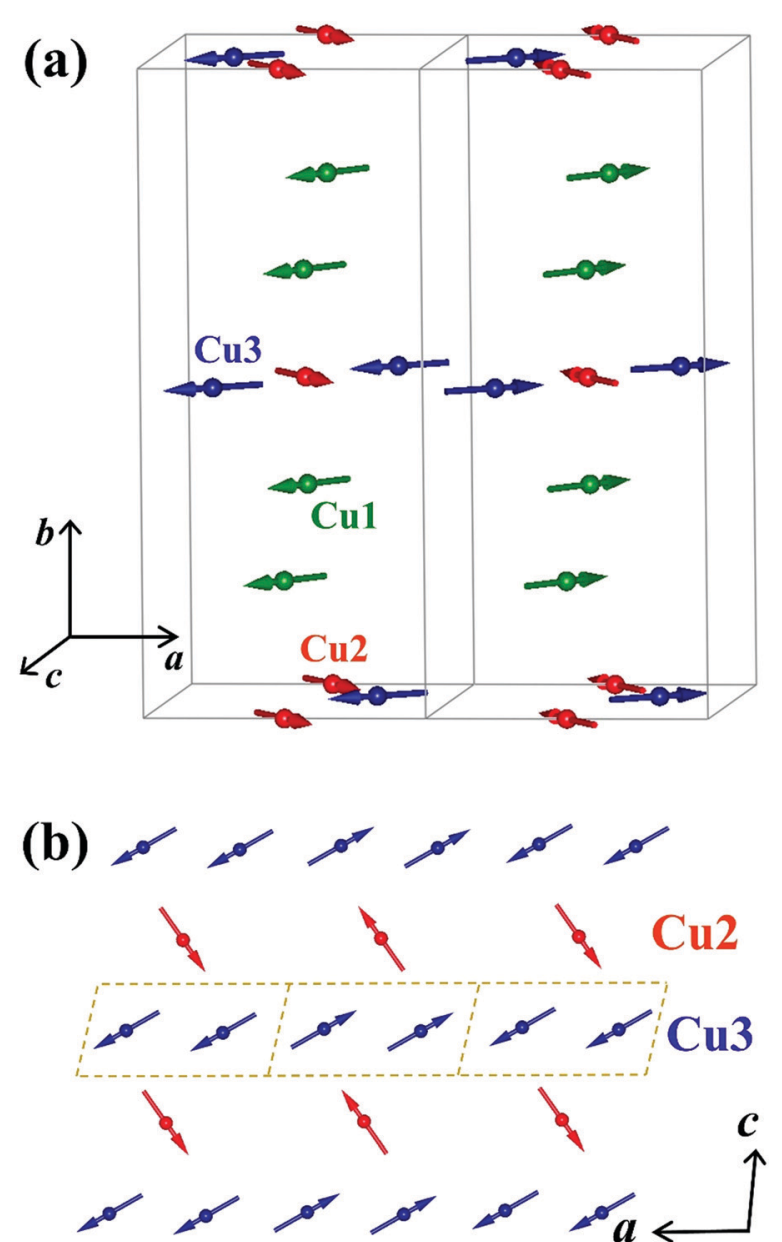

Fig. 6 Schematic drawing of proposed Cu spin arrangements depicting (a) stacking along crystallographic $b$-axis direction and (b) projection of Cu2 and Cu3 spins in crystallographic $a-c$ plane. Dashed rectangular blocks indicate ferromagnetic spin-dimers that formed along a-axis direction. Green, red, and blue arrows indicate moments of Cu1, Cu2 and Cu3 respectively.
Table 3 Magnetic moments of three crystallographically distinct $\mathrm{Cu}$ ions, obtained assuming magnetic space group $P 2_{1} / c$, where $\varphi$ indicates the angle between the moment direction and crystallographic a-axis direction

\begin{tabular}{lrllll}
\hline Atom & $m_{a}\left(\mu_{\mathrm{B}}\right)$ & $m_{b}\left(\mu_{\mathrm{B}}\right)$ & $m_{c}\left(\mu_{\mathrm{B}}\right)$ & $m\left(\mu_{\mathrm{B}}\right)$ & $\varphi$ \\
\hline Cu1 & $-0.48(3)$ & 0 & $0.54(3)$ & $0.76(4)$ & $135^{\circ}$ \\
Cu2 & $0.50(3)$ & 0 & $0.63(4)$ & $0.76(5)$ & $55^{\circ}$ \\
Cu3 & $-0.63(4)$ & 0 & $0.37(3)$ & $0.76(5)$ & $150^{\circ}$
\end{tabular}

$\varphi=$ angle between $m$ and $a$-axis.

this phenomenon deserves further investigation. The Cu1 spins, on the other hand, formed antiferromagnetically coupled ferromagnetic chains along the crystallographic $b$-axis direction. The magnetic moments and their components along the three crystallographic axis directions of the three distinct $\mathrm{Cu}$ ions are presented in Table 3. The antiferromagnetic transition $T_{\mathrm{N}}$ was determined by measuring the thermal variations of the $\left(\begin{array}{lll}1 / 2 & 0 & 0\end{array}\right)$ intensity $I(T)$, as shown in Fig. 5(b), revealing the variation in the square of the magnetization with temperature. Interestingly, $I(T)$ can be expressed as $I=I_{0}\left(1-\frac{T}{T_{\mathrm{N}}}\right)^{2 \beta}$, where $I_{0}$ denotes the saturation magnetic intensity, and $\beta$ the critical exponent of the magnetic transition, with $T_{\mathrm{N}}=32.2(3) \mathrm{K}$ and $\beta=0.21(2)$ [solid curve in Fig. 5(b)]. The obtained critical exponent $\beta=0.21(2)$ is similar to that of the well-known $2 \mathrm{D} \mathrm{La}_{0.6} \mathrm{Ca}_{0.4} \mathrm{MnO}_{3}(\beta=0.25)$ magnet, whereas it is significantly smaller than 0.5 , which is anticipated for a three-dimensional magnetic ordering, which signifies dominant magnetic interactions in $\alpha-\mathrm{Cu}_{5} \mathrm{O}_{2}\left(\mathrm{SeO}_{3}\right)_{2} \mathrm{Cl}_{2}$ occurring in the $2 \mathrm{D}$ plane. ${ }^{47}$ The $T_{\mathrm{N}}=32.2 \mathrm{~K}$ obtained from the magnetic diffraction intensity agreed well with the $T_{\mathrm{N}}$ values determined from $\chi(T)$ [see Fig. 3(a)] and $C / T(T)$ [see Fig. 4(b)].

\subsection{Low-temperature dielectric and magnetodielectric properties}

The low temperature $\varepsilon^{\prime}$ is illustrated in the inset of Fig. 7(a). It exhibited an apparent anomaly near $T_{\mathrm{N}}$. To further investigate the magnetoelectric coupling, an isothermal MD (\%) versus $H$ curve at different temperatures was plotted, as shown in Fig. 7(b). A quadratic nature with a maximum MD value of $0.006 \%$ at $7 \mathrm{~T}(15 \mathrm{~K})$ was indicated, and this quadratic nature was valid even at $25 \mathrm{~K}$; this further confirmed the weak magnetoelectric coupling below $T_{\mathrm{N}}$. Above $T_{\mathrm{N}}$, MD was almost absent. The scaling relation of $\mathrm{MD}(\%) \propto M^{2}$ at 15 and $25 \mathrm{~K}$ [see Fig. 7(c)] shows magnetoelectric coupling, which was streamed from spin-pair correlation of antiferromagnetic systems below $T_{\mathrm{N}}{ }^{48}$ Moreover, the dielectric anomaly in the temperaturedependent $\varepsilon^{\prime}$ for the $\alpha-\mathrm{Cu}_{5} \mathrm{O}_{2}\left(\mathrm{SeO}_{3}\right)_{2} \mathrm{Cl}_{2}$ polycrystalline [shown in Fig. S2, ESI $\dagger$ ] consistent with the Fig. 7(a). The possible answer for the observed magnetoelectric coupling below $T_{\mathrm{N}}$ might be linked to the spin structure. Fig. 6 indicates that the $\mathrm{Cu}^{2+} \mathrm{S}=1 / 2$ spins were frustrated at bow-tie lattice in the $a-c$ plane. The cantings of the $\mathrm{Cu} 2$ and $\mathrm{Cu} 3$ spins in the $a-c$ plane might invoke spin-induced magnetoelectric coupling below $T_{\mathrm{N}}$. Though the centrosymmetric space group down to lowest temperatures usually rules out the crystal symmetry induced magnetoelectric ordering. Furthermore, DM interaction of the 


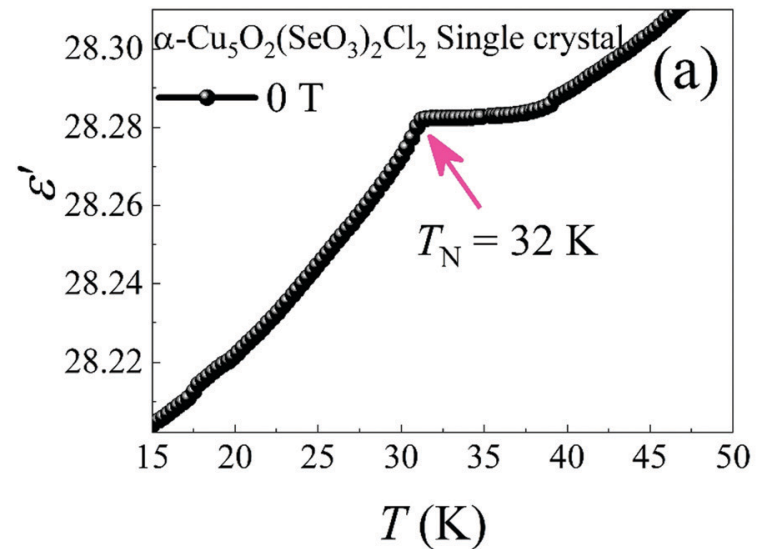

$a$-axis direction shown in rectangular block in Fig. 6(b) satisfy the up-up down-down spin structure. To examine the spininduced electric polarization of $\alpha-\mathrm{Cu}_{5} \mathrm{O}_{2}\left(\mathrm{SeO}_{3}\right)_{2} \mathrm{Cl}_{2}$, temperaturedependent pyrocurrent measurements were performed (the data was not shown here) with a poling electric field of $E=$ $0.4 \mathrm{kV} \mathrm{mm}^{-1}$ was applied during the cooling of the sample from $T=60 \mathrm{~K}$ (well above the dielectric anomaly $T_{\mathrm{N}}=32 \mathrm{~K}$ ). However, pyrocurrent data doesn't exhibit a consistent result for different
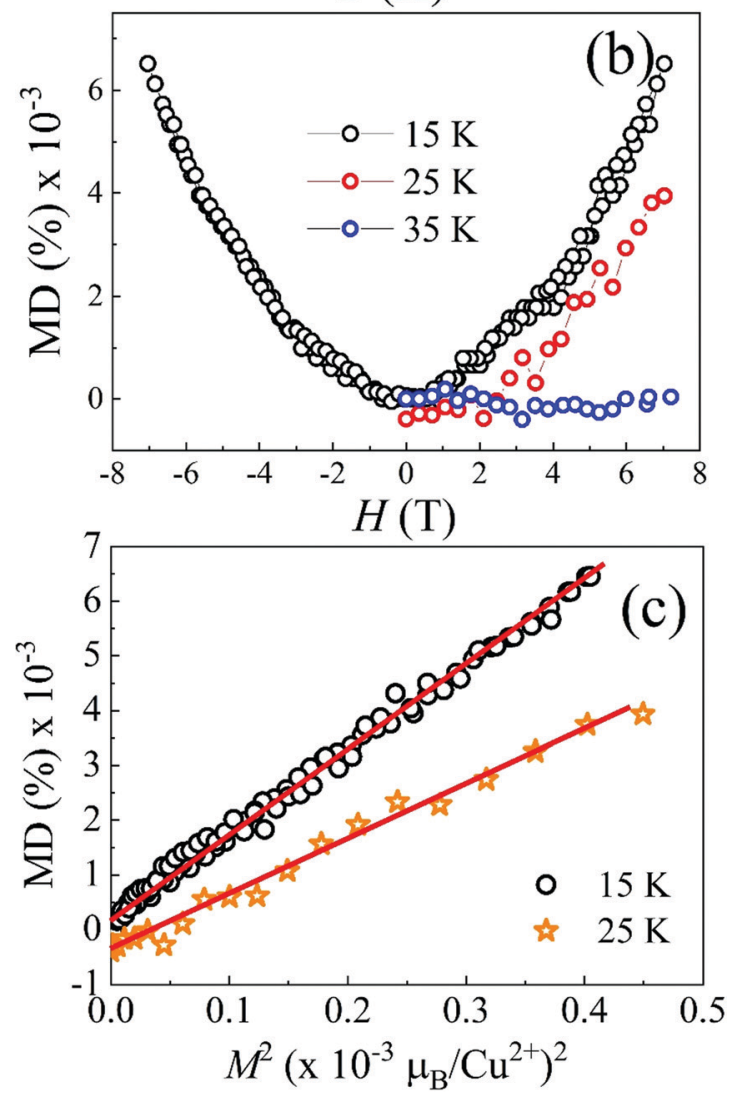

Fig. 7 (a) $\varepsilon^{\prime}$ vs. $T$ curves at low temperature (15-40 K) and $1 \mathrm{MHz}$ for $\alpha-\mathrm{Cu}_{5} \mathrm{O}_{2}\left(\mathrm{SeO}_{3}\right)_{2} \mathrm{Cl}_{2}$ single crystal for $H=0 \mathrm{~T}$. (b) $\mathrm{MD}$ vs. $H$ curves at different temperatures. (c) Shows MD (\%) $\propto M^{2}$ scaling relation for 15 and $25 \mathrm{~K}$, respectively.

form $(\mathrm{S} i \times \mathrm{S} j)$ will be cancelled out for the spin structure of $\alpha-\mathrm{Cu}_{5} \mathrm{O}_{2}\left(\mathrm{SeO}_{3}\right)_{2} \mathrm{Cl}_{2}$ (Fig. 6(a)). However, the possible spininduced magnetoelectric mechanism might be resulted from the exchange-striction of Cu3 spin chains, the up-up and down-down $\mathrm{Cu} 3$ spins along the $a$-axis might be possible invoke magnetoelectric coupling. The up-up down-down spin structure is a well-known spin arrangement in the field of multiferroics. It has predicted theoretically and proved experimentally in several multiferroic systems that the exchange-striction in this spin configuration can induce electrical polarization. ${ }^{49}$ The ferrromagnetic spin-dimers along the
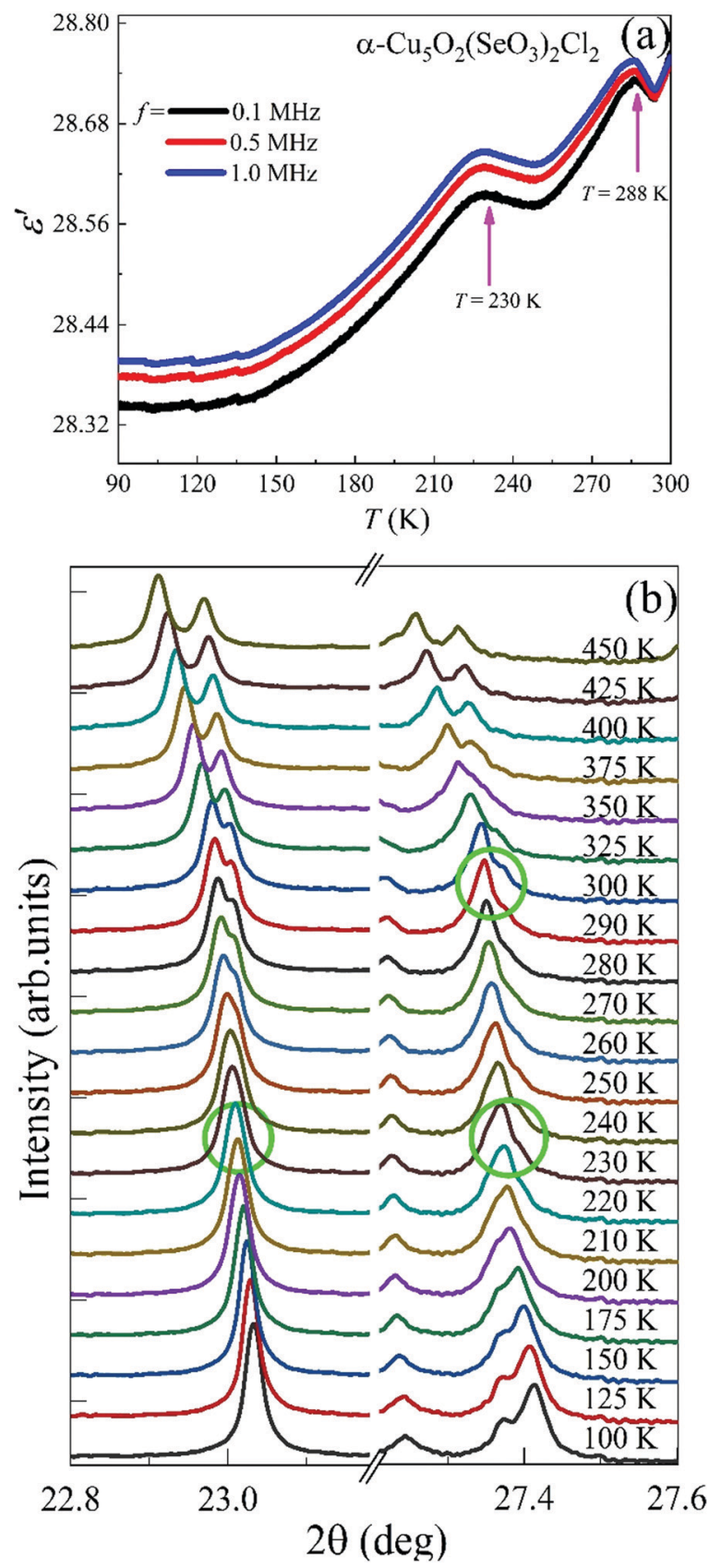

Fig. 8 (a) $\varepsilon^{\prime}$ vs. $T$ curves at high temperature $(90-300 \quad \mathrm{~K})$ for $\alpha-\mathrm{Cu}_{5} \mathrm{O}_{2}\left(\mathrm{SeO}_{3}\right)_{2} \mathrm{Cl}_{2}$ single crystals at various frequencies. (b) Temperature dependencies of SXRD patterns for selected $2 \theta$ ranges. Peaks merged (at $T=290 \mathrm{~K} ; 2 \theta=27.4^{\circ}-27.6^{\circ}$ and at $T=230 \mathrm{~K} ; 2 \theta=22.8^{\circ}-23.2^{\circ}$ ) and split (at $T=230 \mathrm{~K} ; 2 \theta=27.4^{\circ}-27.6^{\circ}$ ); the circles indicate the temperature range where the peaks merged. 
runs, which indicates the present data was not sufficient enough to confirm the electrical ordering. Further studies are needed to give clear picture about the spin-induced electrical ordering, which is out of scope of this paper.

\subsection{High-temperature dielectric and SXRD studies}

The high-temperature $\varepsilon^{\prime}$ versus $T$ curves of $\alpha-\mathrm{Cu}_{5} \mathrm{O}_{2}\left(\mathrm{SeO}_{3}\right)_{2} \mathrm{Cl}_{2}$ measured at $H=0$ [see Fig. 8(a)] showed frequency-independent peaks at $T=288$ and $230 \mathrm{~K}$. These two temperatures are closely associated with the weak specific heat anomalies in the upper inset of Fig. 4(a). However, no clear anomalies were observed in the $\chi$ versus $T$ curves, indicating that these dielectric transitions were not related to the magnetic origin. To investigate the origin of the specific heat and dielectric anomalies at high temperatures, we employed temperature-dependent SXRD from 450 to $100 \mathrm{~K}$. The SXRD data and Rietveld refinement patterns at 450 and $100 \mathrm{~K}$ are shown in Fig. S3 (ESI $\dagger$ ). Both the patterns fit well with the $P 2_{1} / c$ space group, and the obtained refined parameters were satisfactory. The obtained temperaturedependent lattice parameters and the unit cell volume [see Fig. S4, ESI $\dagger$ ] further indicated the weak but noticeable anomalies at 230 and $280 \mathrm{~K}$, and these local structural changes were reflected as anomalies in the specific heat [see the inset of Fig. 4(a)] and dielectric measurements. Fig. 8(b) depicts the SXRD patterns $\left(2 \theta=22.8^{\circ}-23.1^{\circ}\right.$ and $\left.27.2^{\circ}-27.6^{\circ}\right)$ in detail clearly, the merging and splitting of the Bragg reflections occurred at approximately 230 and $280 \mathrm{~K}$, respectively.

In order to investigate the high-temperature structural change, we have analyzed the detailed structural parameters, such as the temperature-dependent average bond lengths of $\mathrm{Cu} 1 \mathrm{Cu} 2$ and $\mathrm{Cu} 3$ polyhedral along with distances within and between the $\langle\mathrm{Cu} 3-\mathrm{Cu} 3\rangle$ spin-chains. As shown in Fig. 9(a), some clear distortions in the $\mathrm{Cu} 1, \mathrm{Cu} 2$ and $\mathrm{Cu} 3$ polyhedral were observed near at $280 \mathrm{~K}$ and $230 \mathrm{~K}$. These structural relaxations of $\mathrm{Cu}$ polyhedral might trigger the structural transitions at these temperatures. Apart from these, the Cu3 spin-chain along the $a$-axis showed the changes within the spin-dimer distances and between the spin-dimers. More interestingly, it showed the formation of strong spin-dimers by decreasing the intra spin-dimer and increasing the inter spin-dimer distance as the temperature was lowered.

The $\mathrm{Cu}^{2+}$ spin-1/2 in Cu-containing oxides exhibits frustrated magnetism, originating either from either the lattice geometry or the dimensional restriction of the magnetic ions. This has resulted in fascinating properties such as multiferroic, magneto-

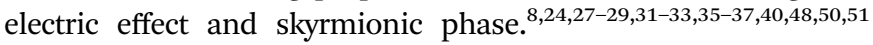
Lone pair $\mathrm{Se}^{4+}$ ions and electron negative halide atoms serve as chemical scissors that can stabilize the low-dimensional magnetic lattice with spin frustration. ${ }^{12}$ Several compounds in the CuO$\mathrm{CuCl}_{2}-\mathrm{SeO}_{2}$ ternary phase diagram were identified and analyzed
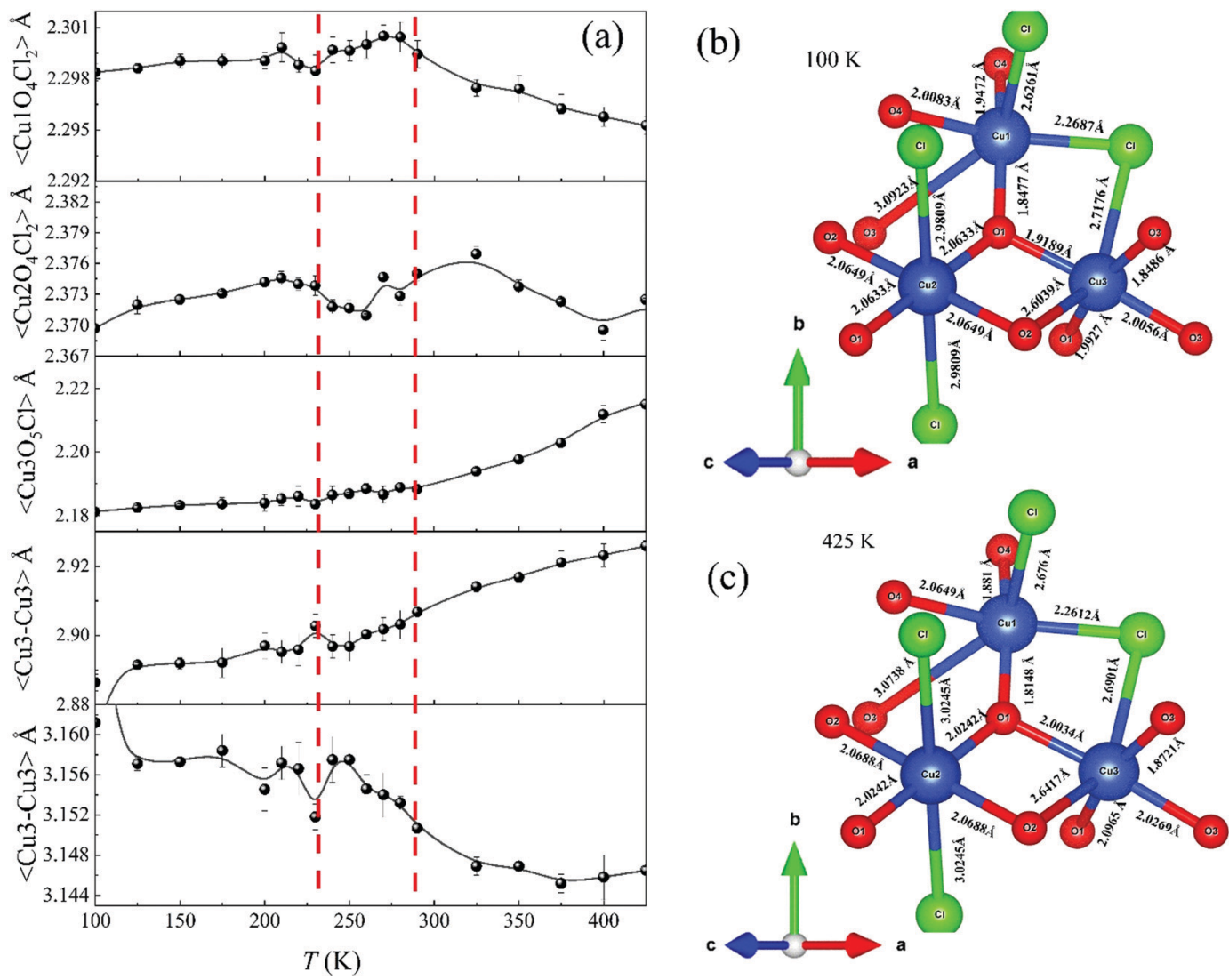

Fig. 9 (a) The temperature variation of average bond lengths of $\left\langle\left\langle\mathrm{Cu}_{1} \mathrm{O}_{4} \mathrm{Cl}_{2}\right\rangle,\left\langle\mathrm{Cu} 1 \mathrm{O}_{4} \mathrm{Cl}_{2}\right\rangle\right.$ and $\left\langle\mathrm{Cu} 3 \mathrm{O}_{5} \mathrm{Cl}\right\rangle$ polyhedral along with distance within and between the Cu3-Cu3 spin-dimers. (b) and (c) Figures show the Cu polyhedral bonds at 100 and $300 \mathrm{~K}$ respectively. 
(see Table 1). Among them, CuO is known as a type-II multiferroic binary compound that is well established near room temperature. ${ }^{27,32}$ High-pressure synthesized $\mathrm{CuSeO}_{3}$ exhibited ferromagnetic ordering at $T_{\mathrm{C}}=25 \mathrm{~K}$, along with a spin-induced dielectric anomaly in a nonpolar state. ${ }^{48}$ The most exciting compound, $\mathrm{Cu}_{2} \mathrm{OSeO}_{3}$, with a $\mathrm{B} 20$ chiral magnetic oxide, exhibited a unique magnetic skyrmion spin. ${ }^{8,30,37} \mathrm{CuCl}_{2}$ was the first reported nonchalcogen in the multiferroic family, where the $\mathrm{Cu}^{2+}$ spin spiral invoked spin-induced robust multiferroicity at $T_{\mathrm{N}}=24 \mathrm{~K}$, even under the significant effect of quantum fluctuation. ${ }^{24}$ Furthermore, multiferroicity was reported in oxyhalide $\mathrm{Cu}_{2} \mathrm{OCl}_{2}$ compounds near the liquid nitrogen temperature, ${ }^{31}$ where two possible mechanisms, namely spininduced or charge-transfer-induced multiferroicity, were proposed. ${ }^{31,35}$ Type-I multiferroic properties have recently been identified in $\mathrm{Cu}_{9} \mathrm{O}_{2}\left(\mathrm{SeO}_{3}\right)_{4} \mathrm{Cl}_{6}$ single crystals. ${ }^{36}$ The $\alpha-\mathrm{Cu}_{5} \mathrm{O}_{2}$ $\left(\mathrm{SeO}_{3}\right)_{2} \mathrm{Cl}_{2}$ in this present study is another magnetoelectric member from the $\mathrm{CuO}-\mathrm{CuCl}_{2}-\mathrm{SeO}_{2}$ phase diagram.

\section{Conclusions}

We successfully synthesized $\alpha-\mathrm{Cu}_{5} \mathrm{O}_{2}\left(\mathrm{SeO}_{3}\right)_{2} \mathrm{Cl}_{2}$ single crystals using the chemical vapor transport method and investigated their structural, magnetic, and dielectric behaviors. The critical findings of this study are as follows: (1) the combined magnetization and specific heat data revealed long-range order at $T_{\mathrm{N}}=32 \mathrm{~K}$. (2) The neutron diffraction patterns confirmed antiferromagnetic ordering below $T_{\mathrm{N}}$ with complex, anisotropic, and two-dimensional spin structure. (3) The high-resolution SXRD data were consistent with the structural changes, which induced two dielectric peaks at $230 \mathrm{~K}$ and $288 \mathrm{~K}$. (4) Observations of weak magnetodielectric effect below $T_{\mathrm{N}}$ suggested a possible spin-induced magnetoelectric behavior in $\alpha-\mathrm{Cu}_{5} \mathrm{O}_{2}\left(\mathrm{SeO}_{3}\right)_{2} \mathrm{Cl}_{2}$. (5) This study point that the bow-tie lattice of $\alpha-\mathrm{Cu}_{5} \mathrm{O}_{2}\left(\mathrm{SeO}_{3}\right)_{2} \mathrm{Cl}_{2}$ is a new spin-frustrated magnetoelectric material.

\section{Author contributions}

H. D. Y., D. C. K., and H. C. W. initiated and designed experiments. Z. H. Y., H. C. W., and D. C. K. synthesized single and polycrystalline samples. C. A. L. and M. C. C. provided the experimental facility for single crystal growth and shared the critical knowledge to grow a good quality single crystal. D. C. K., Z. H. Y., and T. W. K. performed structural characterization and sample quality analysis by synchrotron XRD and EPMA measurements. C. K. C. and B. H. C. helped to perform temperature-dependent synchrotron XRD in NSRRC, Taiwan. J. Y. L. and Y. Y. W. performed the heat capacity measurements. W. H. L., C. H. L. and C. W. W. conducted the neutron diffraction measurements and solved low temperature spin structure. D. C. K., H. C. W., Z. H. Y., and A. T. performed magnetic, dielectric, and magnetodielectric measurements. D. C. K. and H. D. Y. wrote the manuscript by integrating input data and analyses provided by all the authors. H. D. Y. and D. C. K. supervised the research.

\section{Conflicts of interest}

The authors declare no conflict of interest.

\section{Acknowledgements}

This work was supported by the Ministry of Science and Technology, Taiwan, under grant no. MOST 109-2112-M-110019, MOST 108-2112-M-110-014-MY2, MOST 107-2119-M-009 and MOST 107-2917-I-564-008.

\section{References}

1 N. A. Spaldin and R. Ramesh, Nat. Mater., 2019, 18, 203-212.

2 Y. Tokura, S. Seki and N. Nagaosa, Rep. Prog. Phys., 2014, 77, 076501.

3 S. W. Huang, D. J. Huang, J. Okamoto, C. Y. Mou, W. B. Wu, K. W. Yeh, C. L. Chen, M. K. Wu, H. C. Hsu, F. C. Chou and C. T. Chen, Phys. Rev. Lett., 2008, 101, 077205.

4 T. Arima, J. Phys. Soc. Jpn., 2011, 80, 052001.

5 J. S. Kinyon, R. Clark, N. S. Dalal, E. S. Choi and H. Zhou, Phys. Rev. B: Condens. Matter Mater. Phys., 2015, 92, 144103.

6 K. Yoo, B. Koteswararao, J. Kang, A. Shahee, W. Nam, F. F. Balakirev, V. S. Zapf, N. Harrison, A. Guda, N. TerOganessian and K. H. Kim, npj Quantum Mater., 2018, 3, 45.

7 K. Kimura, S. Kimura and T. Kimura, J. Phys. Soc. Jpn., 2019, 88, 093707.

8 S. Seki, X. Z. Yu, S. Ishiwata and Y. Tokura, Science, 2012, 336, 198.

9 B. Keimer, S. A. Kivelson, M. R. Norman, S. Uchida and J. Zaanen, Nature, 2015, 518, 179-186.

10 X. M. Chen, C. Mazzoli, Y. Cao, V. Thampy, A. M. Barbour, W. Hu, M. Lu, T. A. Assefa, H. Miao, G. Fabbris, G. D. Gu, J. M. Tranquada, M. P. M. Dean, S. B. Wilkins and I. K. Robinson, Nat. Commun., 2019, 10, 1435.

11 D. S. Inosov, Adv. Phys., 2018, 67, 149-252.

12 P. S. Berdonosov, E. S. Kuznetsova and V. A. Dolgikh, Crystals, 2018, 8, 159.

13 A. Narayan, A. Cano, A. V. Balatsky and N. A. Spaldin, Nat. Mater., 2019, 18, 223-228.

14 B. Koteswararao, K. Yoo, F. C. Chou and K. H. Kim, APL Mater., 2016, 4, 036101.

15 J. E. Greedan, J. Mater. Chem., 2001, 11, 37-53.

16 P. Puphal, M. Bolte, D. Sheptyakov, A. Pustogow, K. Kliemt, M. Dressel, M. Baenitz and C. Krellner, J. Mater. Chem. C, 2017, 5, 2629-2635.

17 S.-Y. Zhang, W.-B. Guo, M. Yang, Y.-Y. Tang, M.-Y. Cui, N.-N. Wang and Z.-Z. He, Dalton Trans., 2015, 44, 20562-20567.

18 M. Yang, S.-Y. Zhang, W.-B. Guo, Y.-Y. Tang and Z.-Z. He, Dalton Trans., 2015, 44, 15396-15399.

19 Y. Naito, K. Sato, Y. Yasui, Y. Kobayashi, Y. Kobayashi and M. Sato, J. Phys. Soc. Jpn., 2007, 76, 023708.

20 M. Frontzek, J. T. Haraldsen, A. Podlesnyak, M. Matsuda, A. D. Christianson, R. S. Fishman, A. S. Sefat, Y. Qiu, J. R. D. Copley, S. Barilo, S. V. Shiryaev and G. Ehlers, Phys. Rev. B: Condens. Matter Mater. Phys., 2011, 84, 094448. 
21 N. Reynolds, A. Mannig, H. Luetkens, C. Baines, T. Goko, R. Scheuermann, L. Keller, M. Bartkowiak, A. Fujimura, Y. Yasui, C. Niedermayer and J. S. White, Phys. Rev. B, 2019, 99, 214443.

22 J. M. Law, P. Reuvekamp, R. Glaum, C. Lee, J. Kang, M. H. Whangbo and R. K. Kremer, Phys. Rev. B: Condens. Matter Mater. Phys., 2011, 84, 014426.

23 L. Zhao, T.-L. Hung, C.-C. Li, Y.-Y. Chen, M.-K. Wu, R. K. Kremer, M. G. Banks, A. Simon, M.-H. Whangbo, C. Lee, J. S. Kim, I. Kim and K. H. Kim, Adv. Mater., 2012, 24, 2469-2473.

24 S. Seki, T. Kurumaji, S. Ishiwata, H. Matsui, H. Murakawa, Y. Tokunaga, Y. Kaneko, T. Hasegawa and Y. Tokura, Phys. Rev. B: Condens. Matter Mater. Phys., 2010, 82, 064424.

25 S. V. Krivovichev and L. A. Gorelova, Crystals, 2018, 8, 193.

26 H. C. Wu, K. D. Chandrasekhar, J. K. Yuan, J. R. Huang, J. Y. Lin, H. Berger and H. D. Yang, Phys. Rev. B, 2017, 95, 125121.

27 T. Kimura, Y. Sekio, H. Nakamura, T. Siegrist and A. P. Ramirez, Nat. Mater., 2008, 7, 291-294.

28 M. Herak, A. Zorko, D. Arčon, A. Potočnik, M. Klanjšek, J. van Tol, A. Ozarowski and H. Berger, Phys. Rev. B: Condens. Matter Mater. Phys., 2011, 84, 184436.

29 M. Herak, A. Zorko, M. Pregelj, O. Zaharko, G. Posnjak, Z. Jagličić, A. Potočnik, H. Luetkens, J. van Tol, A. Ozarowski, H. Berger and D. Arčon, Phys. Rev. B: Condens. Matter Mater. Phys., 2013, 87, 104413.

30 H. C. Wu, T. Y. Wei, K. D. Chandrasekhar, T. Y. Chen, H. Berger and H. D. Yang, Sci. Rep., 2015, 5, 13579.

31 L. Zhao, M. T. Fernández-Díaz, L. H. Tjeng and A. C. Komarek, Sci. Adv., 2016, 2, e1600353.

32 Z. Wang, N. Qureshi, S. Yasin, A. Mukhin, E. Ressouche, S. Zherlitsyn, Y. Skourski, J. Geshev, V. Ivanov, M. Gospodinov and V. Skumryev, Nat. Commun., 2016, 7, 10295.

33 S. Lee, W. J. Lee, J. van Tol, P. L. Kuhns, A. P. Reyes, H. Berger and K. Y. Choi, Phys. Rev. B, 2017, 95, 054405.

34 H. Guo, L. Zhao, W. Schmidt, M. T. Fernández-Díaz, C. Becker, A. Melendez-Sans, W. Peng, M. Zbiri, P. Hansmann and A. C. Komarek, Phys. Rev. Mater., 2019, 3, 124405.

35 H. C. Wu, J. K. Yuan, K. D. Chandrasekhar, C. H. Lee, W. H. Li, C. W. Wang, J. M. Chen, J. Y. Lin, H. Berger,
T. W. Yen, S. M. Huang, C. W. Chu and H. D. Yang, Mater. Today Phys., 2019, 8, 34-42.

36 H. C. Wu, K. N. Denisova, D. Menzel, D. C. Kakarla, O. V. Maximova, T. W. Kuo, Z. H. Yang, C. H. Lee, W. H. Li, H. Berger, C. W. Wang, C. K. Chang, Y. C. Chuang, J. Y. Lin, M. Gooch, C. W. Chu, A. N. Vasiliev and H. D. Yang, Phys. Rev. B, 2019, 100, 245119.

37 L. Deng, H.-C. Wu, A. P. Litvinchuk, N. F. Q. Yuan, J.-J. Lee, R. Dahal, H. Berger, H.-D. Yang and C.-W. Chu, Proc. Natl. Acad. Sci. U. S. A., 2020, 117, 8783.

38 K. D. Chandrasekhar, H. C. Wu, C. L. Huang and H. D. Yang, J. Mater. Chem. C, 2016, 4, 5270-5274.

39 L. P. Vergasova, S. V. Krivovichev, S. K. Filatov, S. N. Britvin, P. C. Burns and V. V. Anan'ev, Geol. Ore Deposits, 2007, 49, 518-521.

40 S. V. Krivovichev, R. R. Shuvalov, T. E. Semenova and S. K. Filatov, Z. Kristall., 1999, 214, 135-138.

41 J. Galy, J. J. Bonnet and S. Andersson, Acta Chem. Scand., 1979, 33, 383-389.

42 A. J. Studer, M. E. Hagen and T. J. Noakes, Phys. B, 2006, 385, 1013-1015.

43 O. Yakubovich, G. Kiriukhina, O. Dimitrova, A. Volkov, A. Golovanov, O. Volkova, E. Zvereva, S. Baidya, T. SahaDasgupta and A. Vasiliev, Dalton Trans., 2013, 42, 14718-14725.

44 S. E. Dutton, Q. Huang, O. Tchernyshyov, C. L. Broholm and

R. J. Cava, Phys. Rev. B: Condens. Matter Mater. Phys., 2011, 83, 064407.

45 K. Devi Chandrasekhar, J. Krishna Murthy, J. Y. Lin, H. C. Wu, W. J. Tseng, A. Venimadhav and H. D. Yang, Phys. Rev. B, 2016, 94, 205143.

46 J. Rodríguez-Carvajal, Phys. B, 1993, 192, 55-69.

47 D. Kim, B. Revaz, B. L. Zink, F. Hellman, J. J. Rhyne and J. F. Mitchell, Phys. Rev. Lett., 2002, 89, 227202.

48 G. Lawes, A. P. Ramirez, C. M. Varma and M. A. Subramanian, Phys. Rev. Lett., 2003, 91, 257208.

49 S. Picozzi, K. Yamauchi, B. Sanyal, I. A. Sergienko and E. Dagotto, Phys. Rev. Lett., 2007, 99, 227201.

50 S. V. Krivovichev, S. K. Filatov, P. C. Burns and L. P. Vergasova, Can. Mineral., 2007, 45, 929-934.

51 P. Millet, B. Bastide and M. Johnsson, Solid State Commun., 2000, 113, 719-723. 